\title{
Optimization of Elastic Nonlinear Behavior Measurements of Ceramic Piezoelectric Resonators with Burst Excitation
}

\author{
Alfons Albareda, Member, IEEE, Rafel Pérez, Jorge A. Casals, Jose E. García, and Diego A. Ochoa
}

\begin{abstract}
A system of nonlinear measurement and nonlinear elastic characterization of resonators is presented, which increases the possibilities and characteristics of the other classic nonlinear characterization methods. This characterization has been necessary due to the use of resonators in power devices, where their behavior departs from the linear characteristics. The use of burst signals and a system of acquisition and data processing is proposed instead of impedance analyzers, thus avoiding the thermal effects associated with the high-signal measures, which are necessary for this characterization. The measures are repeated for different amplitudes and at the same frequency near the resonance by a single amplitude sweep, which is simpler and faster to carry out than the multiple frequency sweepings used in other methods. As a last resort, a variation on the proposed method, closer to the classical measures, is put forward, in which the resonance is ensured in all the measures. Special emphasis is placed on obtaining nonlinear characterization of the piezoceramic material in order to increase its optimization in the transducers in terms of both its use and its composition and structure.
\end{abstract}

\section{INTRODUCTION}

W HEN piezoelectric devices are used in resonance, if the excitation signal is elevated, as happens in the power devices, an alteration of the linear resonator behavior occurs and nonlinear phenomena appear [1]-[12]. In many cases, this behavior alters the normal operation of devices; thus it is important to have a nonlinear characterization that can foresee what will occur.

This nonlinearity is due to the great strains and stresses that the resonator undergoes in the neighborhood of the resonance, and for this reason we will deal in general with nonlinear elastic behavior. This does not rule out the existence of other piezoelectric and dielectric nonlinear behaviors, which can be superposed to the nonlinear elastic one.

Among the most important nonlinear elastic alterations that may occur in the resonance, the following phenomena that appear when the excitation signal is increased may be emphasized:

- Increase of mechanical losses.

Manuscript received January 24, 2007; accepted May 9, 2007. The work was supported by the Spanish MEC (project MAT2004-01341) and the European Network POLECER (G5RT.CT-2001-05024).

The authors are with the Department of Applied Physics, Universitat Politècnica de Catalunya, Barcelona, Spain (e-mail: alfons@fa.upc.edu).

Digital Object Identifier 10.1109/TUFFC.2007.514
- Amplitude-frequency shift effect.

- Appearance of frequency hysteresis when a frequency sweep at constant voltage is applied. For certain materials and for high amplitudes, the resonance is reached only when the sweep is made in decreasing frequencies.

- Harmonic generation at frequencies different from the excitation signal.

If these nonlinear properties of the materials and the devices are known accurately, the operating limits of the resonators can be determined. It is also important to determine when the frequency hysteresis takes place, because sweepings in decreasing frequencies must be carried out so that the resonance can always be reached [12].

To carry out the material nonlinear characterization, it is necessary to take piezoelectric resonators with suitable forms to be able to characterize the nonlinearity of the relevant elastic tensorial coefficients: $s_{33}, s_{11}$, etc. In the present work, it is assumed that the constitutive equations are valid, but it is accepted that the coefficients cease to be constant and become a function of the excitation level amplitude.

This nonlinear characterization informs us about the material characteristics. In effect, the piezoelectric materials used are usually PZT ceramics, and they present extrinsic behaviors due to the ferroelectric domain wall movements. These extrinsic effects are the main cause of the nonlinear behavior, whereas the intrinsic contribution, which corresponds to a monodomain, is linear [13][20]. Therefore the nonlinear coefficients measure approximately the quantity of the extrinsic effect, thus providing us with information about the ceramic microstructure. Nevertheless, this extrinsic effect, which for their nonlinearity may be better to avoid, is indeed responsible for the fact that these PZT ceramics have such good qualities, both their high piezoelectric coefficient value and their high dielectric permittivity value. Thus the materials that have high piezoelectric coefficient values are often also those that present a higher nonlinearity (for example, soft materials) [19], [21].

A possible nonlinear characterization is made by using the intermodulation method. A nonlinear effect characteristic is the harmonic generation, so signals at frequencies different from the excitation frequency are produced. When two excitation signals at different frequencies are used, the response will contain a combination of the sum and the difference of the excitation signals. This sensitive 
method [22], [23] enables us to obtain the nonlinear characterization with good accuracy, without increasing the excitation signal level excessively. However, this characterization type will not be considered in this work, because this system departs too much from the possible power applications we wish to characterize and does not enable us to measure the vibration velocity at the same time. Furthermore, the results obtained by this method are correct only for very low nonlinearities, and the high-level behavior is not always similar.

Nonlinearity also produces harmonic generation at frequencies different from the excitation signal. These new signals are generally of little importance because their frequencies are far away from the natural resonance and their amplitudes are low, so they do not disturb the normal device operation. In the present work, only the generator frequency signals are measured in order to obtain the dependence of compliance and losses on the amplitude level. However, the system is able to measure the other harmonic signal contributions if required.

An important aspect in nonlinear behavior characterization of piezoelectric resonators is to prevent sample temperature increase when the measurements are made. Application of a high-level signal to a resonator causes an increase in its temperature, so that both phenomena can be superposed: nonlinearity and thermal effects. In order to prevent this, it is necessary to design an appropriate measurement method.

One of the ways of preventing the thermal effects is to take the measurements as rapidly as possible - with an impedance analyzer - at the cost of reducing the accuracy of the measurements. After each measurement it is necessary to wait long enough before taking the next one.

Another way of obtaining this is by the use of burst signals which, since they are more limited in time, prevent overheating. In this work we present the advantages of this characterization system with burst excitation, analyzing to the maximum all of the possibilities and improvements that we have developed in its implementation.

First, we present the different measurement and characterization methods, and later we will adapt these measurement types to the burst signals used. Throughout this work, resonant discs in radial mode have mainly been used, since there are no superposed spurious signals due to other modes, and they are easy to use, although some mathematical aspects are more complex and are detailed in the appendix. References to other resonant modes are made only in some cases. The method developed here will be useful for the characterization of those nonlinear coefficients that correspond to the vibration mode used, and the conclusions presented are generalized to any other vibration mode.

\section{Previous Work: Measurement Types}

The most usual resonator nonlinear characterization consists of the measurement of the mechanical losses in- crease, $\Delta \tan \delta_{m}$, and the resonance frequency decrease, versus the amplitude of the excitation signal [12], [24].

A way of obtaining this characterization is by the resistance measurement $R$ (or inverse of the maximum admittance modulus $\left.\left|Y_{\max }\right|\right)$ at resonance, which is proportional to the losses, and the reactance variation measurement, $\Delta X$, due to the signal level increase. This reactance increase is proportional to the resonance angular frequency decrease $\Delta \omega_{s}$ :

$$
\begin{aligned}
\frac{\Delta R}{\bar{Z}} & =\Delta \tan \delta_{m}=\Delta\left(\frac{1}{Q_{m}}\right)=\Psi\left(I_{m}\right), \\
\frac{\Delta X}{\bar{Z}} & =-2 \frac{\Delta \omega_{s}}{\omega_{s 0}}=\Psi^{\prime}\left(I_{m}\right), \\
\bar{Z} & =L_{m 0} \omega_{s 0}
\end{aligned}
$$

where $L_{m 0}$ is the motional inductance, from the low signal equivalent circuit; $\omega_{s}$ and $\omega_{s 0}$ are the resonant frequencies at high and low signal level; $Q_{m}$ is the mechanical quality factor; and $\Psi$ and $\Psi^{\prime}$ are functions of the motional current $I_{m}$.

In these equations, the mechanical losses and the resonant frequency variations are related to the excitation signal level through the motional current $I_{m}$. This motional current is proportional to the spatial mean strain $\langle S\rangle$ that exists in the resonator, and is the main cause of the nonlinear behavior at these frequencies. This will be the independent magnitude [12] which makes it possible to obtain a good nonlinear elastic characterization.

There are several measurement systems used to make the nonlinear characterization: frequency sweeps at constant voltage or at constant current, or amplitude sweeps at constant frequency. We analyze each of them.

\section{A. Frequency Sweeps at Constant Voltage}

The classical system to carry out the nonlinear characterization is the use of an impedance analyzer making frequency sweeps at different voltage amplitude levels, obtaining for each level the resistance value at resonance and the resonant frequency (Fig. 1). This is the measurement system at "constant voltage." Both the mechanical losses values $\tan \delta_{m}$ and the resonant frequency $f_{s}$ are obtained at each voltage amplitude level of the applied signal [7], [11], [12], [24].

The nonlinear effects are due to the high strains and stresses that appear at resonance, so it is necessary to carry out the current measurement in order to obtain the motional current and the mean strain $\langle S\rangle$ (A15).

This measurement system has been used to study the elastic nonlinearities, and the characterization obtained shows that the nonlinear contributions, both on the mechanical losses and in resonant frequency, depend exclusively on the motional current or mean strain $\langle S\rangle$.

One of the problems of this method is that in these measurements one complete frequency sweep is carried out to obtain only one point on the nonlinear characterization curve. Therefore it is necessary to make as many frequency 


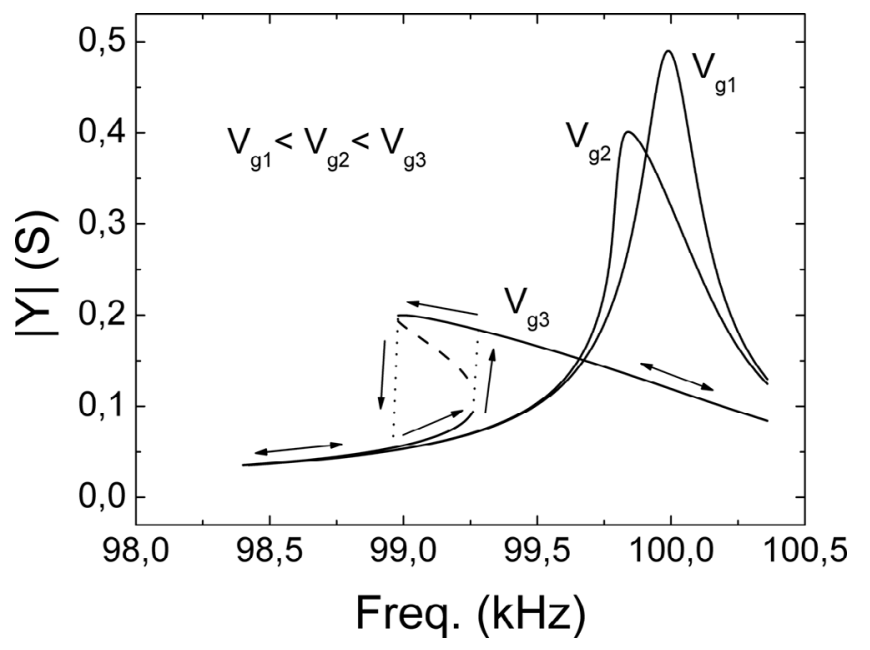

Fig. 1. Admittance modulus representation of resonance for different input levels: frequency hysteresis at high level amplitude.

sweeps as points on this curve, with the corresponding temperature increase. Another problem is that it is necessary to make the frequency sweep only in decreasing frequencies, in order to ensure that the resonance is always reached, even if the hysteresis phenomenon appears. Finally, some analyzers do not enable the impedance and current measurements to be taken simultaneously.

\section{B. Frequency Sweeps at Constant Current}

Hirose [6], [25] has proposed carrying out the measurements at "constant motional current." He uses a feedback electronic system that generates a constant current signal, after compensating the electrical capacitance $C_{0}$ (dielectric capacitance, in parallel with the motional branch), to ensure that the motional current flowing through the resonator remains constant. The main advantage of this system is that the frequency hysteresis disappears, enabling frequency sweeps at increasing or decreasing frequencies to be made.

A drawback of this system is the use of a rather sophisticated, nonstandard generator. However, the main problem of this measurement method is that the power applied to the resonator is always very high. For all the frequencies of a sweep, the power applied is the same as that used only at resonance $f_{s}$ in the previous method.

\section{Amplitude Sweeps at Constant Frequency}

A further measurement method developed in [11], [12] consists of making an amplitude sweep at "constant frequency." The measurements of resistance and reactance increase for the nonlinear characterization depend only on the motional current and not on the frequency. In this system, an amplitude sweep is carried out for a fixed frequency near the resonance, and the measurements of the resistance $R$, reactance $X$, and current $I$ are made in order to obtain the resonator nonlinear characterization. This amplitude sweep is repeated at another close frequency to verify that the nonlinear characterization is similar.

This is a very important point in this work. Only if the curves $R\left(I_{m}\right)$ and $\Delta X\left(I_{m}\right)$ are frequency independent is it possible to obtain the nonlinear laws by this method. Furthermore, in this case, only one amplitude sweep is necessary to complete the nonlinear characterization.

A drawback of this method is that not all impedance analyzers allow these measurements. Some have no amplitude sweep capability available, even if it allows the necessary measures $R, X$, and $I$ (Agilent 4194 impedance analyzer, Agilent Technologies, Inc., Santa Clara, CA). Other analyzers could make these amplitude sweeps, but they do not allow the simultaneous measurements of the current (Agilent 4294). Given these disadvantages, the solution adopted consists of making the voltage and the current measurements by an oscilloscope, using a burst excitation signal explained later. An automatic measurement control system performs the data acquisition, and later performs the data treatment necessary to obtain the nonlinear characterization.

These measurements are made simultaneously with the vibration velocity measurements $v$ on a resonator characteristic point, for example, on an edge point. They could easily be made by the use of a laser vibrometer. The vibration velocity measurements informs us directly of the mean strain $\langle S\rangle$, making the nonlinear measurement of piezoelectric coefficients $d$ and $e$ possible. These laser vibrometer measurements are difficult to carry out simultaneously with the impedance analyzer measures, but they are easily carried out when an oscilloscope performs the data acquisition (it suffices to use a three-channel oscilloscope to obtain the signals $V, I$, and $v$ ).

As set out above, a nonlinear characterization method by means of an amplitude sweep at constant frequency is proposed, with an oscilloscope data acquisition to obtain the voltage $V$, the current $I$, and the vibration velocity $v$, and a subsequent data treatment. Another possibility will be also commented upon, which consists of taking the measurements at resonance instead of at constant frequency, but with only one amplitude sweep.

\section{Burst Measurements: Free Vibrations IN THE TAIL}

As previously stated, an important aspect in the nonlinear characterization at high power is to take the measurements without the resonators overheating [7], [10]. The best solution is the use of burst signals, since this prevents the temperature effects and is perfectly compatible with the use of an oscilloscope to make the measurements, as well as measurement of the vibration velocity at a point of the resonator by a laser vibrometer.

To do this, the first option is the use of a burst with a high signal level and by taking the measurements in the free response at the transient state produced in the tail of the burst, when the excitation signal is finished [Fig. 2(a)] 

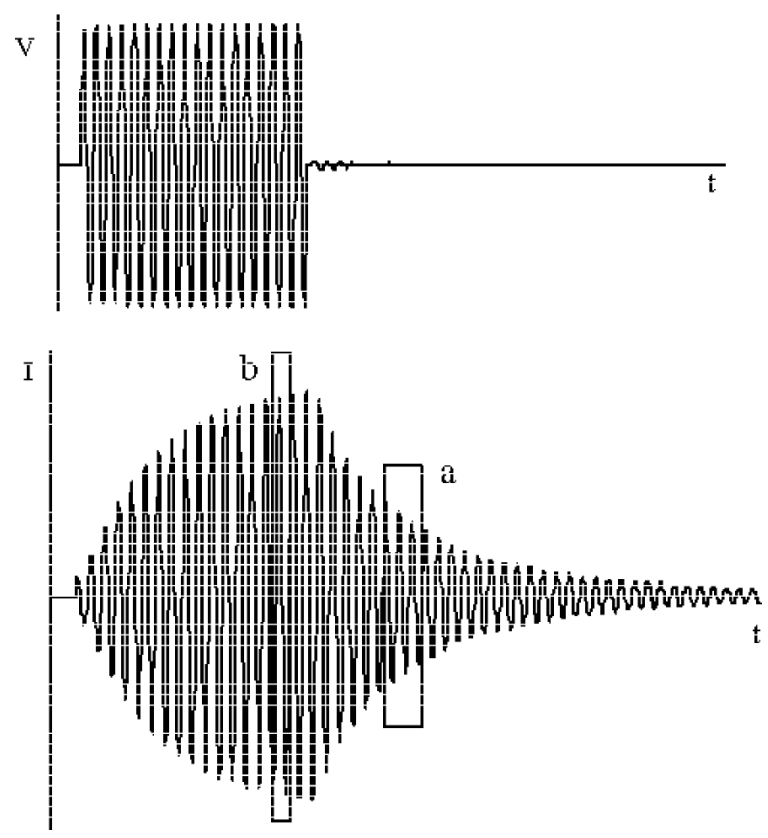

Fig. 2. Voltage and current of the burst signal: (a) free vibrations in the tail; (b) steady-state vibrations before the end of the burst excitation.

[26]-[30]. The time constant $\tau(I)$ is calculated from the enveloping amplitude signal for different current levels $I$, and so for different instants of the burst tail. The oscillation period $T$ and frequency $f$ at the same instants are also calculated. From all of these measurements, the losses $\tan \delta_{m}(I)$, and shift frequency $\Delta f / f_{0}(I)$ versus the current level can be obtained. This measurement system is completed with velocity measures by a laser vibrometer $v(I)$.

To carry out these measurements, an oscilloscope with a data acquisition is necessary. A data treatment system allows us to obtain the enveloping signal from the consecutive maximum points of the damping oscillations. From this decay curve the time constant $\tau(I)$ is calculated. This calculation is made from the logarithmic variation of the current. The measurement of the oscillation period $T$ and the calculation of the resonant frequency $f_{s}(I)$ allow us to obtain the losses $\tan \delta_{m}(I)$ and the shift frequency $\left(f_{s}-f_{s 0}\right)(I)$ for each current level amplitude $I$, where $f_{s 0}$ is the resonant frequency at low level amplitude:

$$
\begin{aligned}
\tan \delta_{m} & =\frac{1}{Q_{m}}=\frac{1}{2 \pi f \tau}, \\
\frac{\Delta f_{s}}{f_{s 0}} & =\frac{f_{s 0}-f_{s}}{f_{s 0}} .
\end{aligned}
$$

This measurement system produces noise, particularly in the velocity measurements taken by the laser vibrometer, because, for example, it does not allow the use of adequate filters to eliminate this noise. To prevent or minimize this noise, it is necessary to use digital filters in the treatment data, and to repeat the measures several times and work out an average.

Measurements of this type have been successfully used [29] to characterize materials with a high mechanical qual- ity factor, working at the first resonance mode, which is that at the lowest frequency. However, when it has been used to characterize 3-1 composites [26]-[28], serious measurement difficulties has been found. Indeed, in this case it is easy to find several resonant modes, for example, thickness and lateral modes, with close frequency values. When the resonator is in the free oscillation interval, at the transient state produced in the tail of the burst, a beat phenomenon may easily occur between the different frequencies, because several vibration modes are excited. These beat effects, which are reflected in the enveloping signal, make the correct time constant measurements impossible.

\section{Burst Measurements: Steady-State Vibration, Constant Frequency}

The solution adopted in this work is the use of burst signal excitation to prevent thermal effects on the resonators, but carrying out the measurements in the time interval of the steady-state vibration zone [Fig. 2(b)]. To this end, after the beginning of the burst excitation, a prudent delay is incorporated during a number of cycles $N_{1}$ to ensure that the transient state is finished and that the steady-state is achieved. At this moment, all signals are captured for a cycle number $N_{2}$, just before the end of the burst excitation signal. During this interval of $N_{2}$ cycles, all signals necessary for making the characterization are obtained: voltage $V(t)$, current $I(t)$, and velocity $v(t)$.

With this acquisition data system it is possible to perform the nonlinear measurements, preventing, for example, the beating phenomenon generated in the piezocomposites: When a work frequency is imposed, these beats are avoided, because the undesired modes are not excited. At the same time, it is possible to use acquisition measurements at generator frequency, which enables noise to be eliminated.

In the experimental system developed, a four-channel oscilloscope allows us to obtain the signals $V(t), I(t)$, and $v(t)$ during $N_{2}$ cycles of period $T$. The cycle number value $N_{1}$ necessary to reach the steady state is a function of the mechanical quality factor $Q_{m}$ of the resonator. The value of $N_{1}=3 \cdot Q_{m}$ has been accepted. After the acquisition of these signals, they are multiplied by the sine and cosine functions at the same generator frequency $\omega$, and integrated during a time $N_{2} T$. This method prevents the noise and obtains the relative phase between signals: Exclusively, measurements of the fundamental harmonic component are made, similar to those carried out in a lockin amplifier, by a synchronous detection.

If we suppose that the voltage is $V_{0} \cos \left(\omega t+\varphi_{v}\right)$, and that there are noise signals at other $\omega^{\prime}$ frequencies, the average of the product with the sine and cosine functions during an entire number $N_{2}$ of periods will be

$$
\frac{1}{N_{2} T} \int_{t_{1}}^{t_{1}+N_{2} T} V_{0} \cos \left(\omega t+\varphi_{v}\right) \cos (\omega t) d t=\frac{1}{2} V_{0} \cos \left(\varphi_{v}\right)
$$




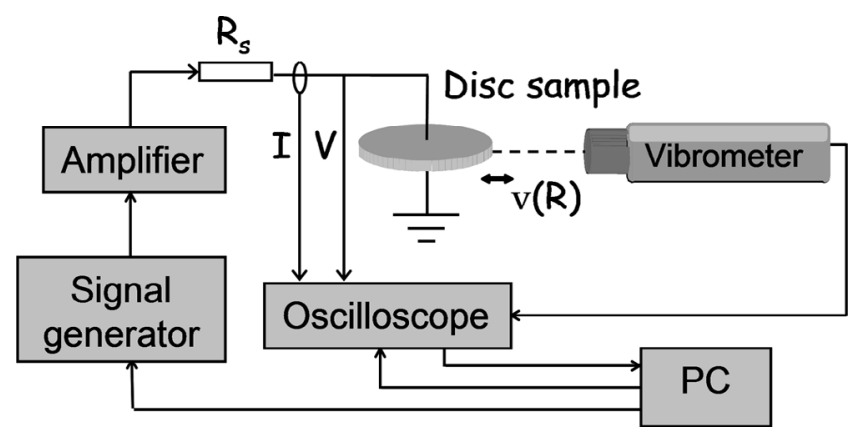

Fig. 3. Experimental block diagram.

$$
\frac{1}{N_{2} T} \int_{t_{1}}^{t_{1}+N_{2} T} V_{0} \cos \left(\omega t+\varphi_{v}\right) \sin (\omega t) d t=-\frac{1}{2} V_{0} \sin \left(\varphi_{v}\right)
$$

The result of this integration is zero for all noise signals with frequencies $\omega^{\prime}$ different from the $\omega$ frequency of the sine and cosine signals.

These results will constitute the real and imaginary part of the complex $\boldsymbol{V}$. Calculating in a similar way with the current $\boldsymbol{I}$ and the velocity $\boldsymbol{v}$, this method allows us to construct the complex impedance $\boldsymbol{Z}=\boldsymbol{V} / \boldsymbol{I}=R+j X$. In a first step, the nonlinear behavior can be obtained from the dependences $R(I), X(I)$, and $v(I)$ to arrive at the nonlinear coefficient and dependence laws by a data treatment.

This measurement process can be performed for a small number of cycles, repeating the process several times and making an average in the oscilloscope. However, this implies that several long burst signals are applied for each measure, but using only these few numbers of cycles. Finally, the solution adopted consists of using a large number of $N_{2}$ cycles, for example, $N_{2}=100$ or 500 , employing only one burst signal. This system avoids the use of an average of several signals and optimizes the efficacy of the burst signal in relation to the information obtained. Consequently, a lower thermal effect is produced on the resonator.

\section{EXPERIMENTAL System}

The excitation measurement system is composed of a generator (HP 33120A, Agilent Technologies, Inc.) and an amplifier (NF 4025, NF Electronic Instruments, Yokohama, Japan) with low output impedance $(0.1 \Omega)$ and high maximum frequency $(2 \mathrm{MHz})$, well adapted to the resonance measurements requirements (Fig. 3). The velocity signal $v(t)$ is obtained by a vibrometer laser (Polytec OFV 3001/303, Polytec GnbH, Waldbronn, Germany). A fourchannel digital oscilloscope (LeCroy 6030, LeCroy Corporation, Chestnut Ridge, NY)) provides the time dependent signals of voltage $V(t)$, current $I(t)$ (by a Tektronix CT2 current probe, Tektronix Espanola SA, Madrid, Spain), and velocity $v(t)$.

The data acquisition and treatment system (HP-VEE, Hewlett-Packard Espanola, Madrid, Spain) has the follow- ing important aspects, which are pointed out schematically:

- It controls the generator: fixes the frequency and amplitude of the burst signal and the number of cycles. A single burst is used to make a measurement point. A typical measurement cycle contains several amplitude levels (16 different values) for each frequency (3 or 6 different values) near the resonance. These measurements allow us to verify that the nonlinear characterization is frequency independent, with a good approximation.

- It controls the oscilloscope: the delay time of $N_{1}$ periods, after the beginning of the external signal of the burst generator. Special attention has been given to the vertical scale for each channel: It is programmed to provide the optimum scale for each channel, taking into account the previous measurements and the consecutive adjustments, in order to obtain a signal without exceeding the oscilloscope limits, but with the maximum of information, with the aim of covering between $70-80 \%$ of the scale maximum. That is to say, an automatic vertical scale generation has been carried out. The oscilloscope sampling frequency is about 100 times the resonant frequency.

- An estimation of the resonator maximum temperature overheating is obtained. From the material heat specific and dimensions, the heat capacity and the temperature maximum increase is calculated, starting from the measured voltage and current and from the cycle number and frequency of the burst. It is assumed that the resonator always works in the steady state and that there is no radiation heat, so a maximum value of the temperature increase is obtained. The process is accumulative for all of the measurements.

- From the imposed data of excitation signal frequency, the signals $\sin (\omega t)$ and $\cos (\omega t)$ are generated, to be used in the previously mentioned measurements at generator frequency (6), (7). As previously explained (Section IV), the complex voltage $\boldsymbol{V}$, current $\boldsymbol{I}$, and velocity $\boldsymbol{v}$ are obtained from the oscilloscope signals $V(t), I(t)$, and $v(t)$, respectively. Finally, the complex impedance $\boldsymbol{Z}=\boldsymbol{V} / \boldsymbol{I}=R+j X$, is calculated.

- A calibration is necessary to correct the phase induced by the whole system between the signals.

- After the research data $R, X, I, v$, and $f$, are obtained, they are transferred to a spreadsheet for later data treatment.

\section{Nonlinear Characterization}

\section{A. Motional Impedance Plane}

To make the nonlinear characterization, it is necessary to transform the direct measurements to other more appropriate magnitudes. First, since the nonlinearity is associated to the mechanical or motional parameters of the res- 
onator, it is necessary to modify the magnitudes $R$ and $X$ for the corresponding motional magnitudes $R_{m}$ and $X_{m}$, which the resonator would have if the dielectric contribution $C_{0}^{p}$ were disconnected. This capacitance $C_{0}^{p}$ corresponds to the dielectric contribution in the frequency interval between the radial resonance and the thickness resonance, for the disc resonators (see Appendix):

$$
R_{m}+j X_{m}=Z_{m}=\frac{1}{Y_{m}}
$$

where

$$
Y_{m}=Y-Y^{P}=\frac{1}{Z}-Y^{P}
$$

and

$$
Y^{P}=j \omega C_{0}^{P}
$$

For other vibration modes this capacitance will be taken similarly.

In the same way, the total current $I$ allows the calculation of the motional current $I_{m}$, to be used as the independent variable. In the second step, it is necessary to find a magnitude that is independent of the resonator dimension. A first possibility is the use of the electric displacement $D_{m}=I_{m} / A \omega$ corresponding to the motional current, which is directly proportional to the mean strain $\langle S\rangle$. An important question is whether the mean strain $\langle S\rangle$ or its time derivative $\langle d S / d t\rangle$ is the best magnitude; that is to say, whether magnitude $D_{m}$ or $d D m / d t$ is better. To this end, nonlinear characterization of resonators with the same material and different dimensions has been carried out [12]. It is observed that the best magnitude to explain the nonlinearity is $\langle S\rangle$ or $D_{m}$, because the dependence of the nonlinear parameters on the resonator frequency is much lower than if the magnitudes $\langle d S / d t\rangle$ or $d D_{m} / d t$ are used.

It is important to remark on the dependence on the mean strain $\langle S\rangle$. Uchino and Hirose [6], [7], [25] analyzed the nonlinear behavior of resonators, taking as the independent magnitude the velocity $v$ in a resonator edge point. For a bar resonator in longitudinal mode, the relation between these magnitudes $v$ and $\langle S\rangle$ is

$$
v=\frac{\langle S\rangle \omega_{s} L}{2}=\frac{\langle S\rangle \omega_{s} \lambda}{4}=\frac{\langle S\rangle c \pi}{2},
$$

where $L$ is the bar longitude, and $c$ is the acoustic wave propagation velocity, which is near constant, with low variations for different materials. For other resonant modes, the frequency is always proportional to the inverse of the dimension associated to the resonator mode $\omega \alpha 1 / L$.

Thus the velocity vibration $v$ and the main strain $\langle S\rangle$ are proportional magnitudes, and are equivalent to taking any of them as the independent magnitude. The distinction is only qualitative, whereas the vibration velocity is important in applications, and more especially in resonant devices. On the other hand, the mean strain $\langle S\rangle$ and the

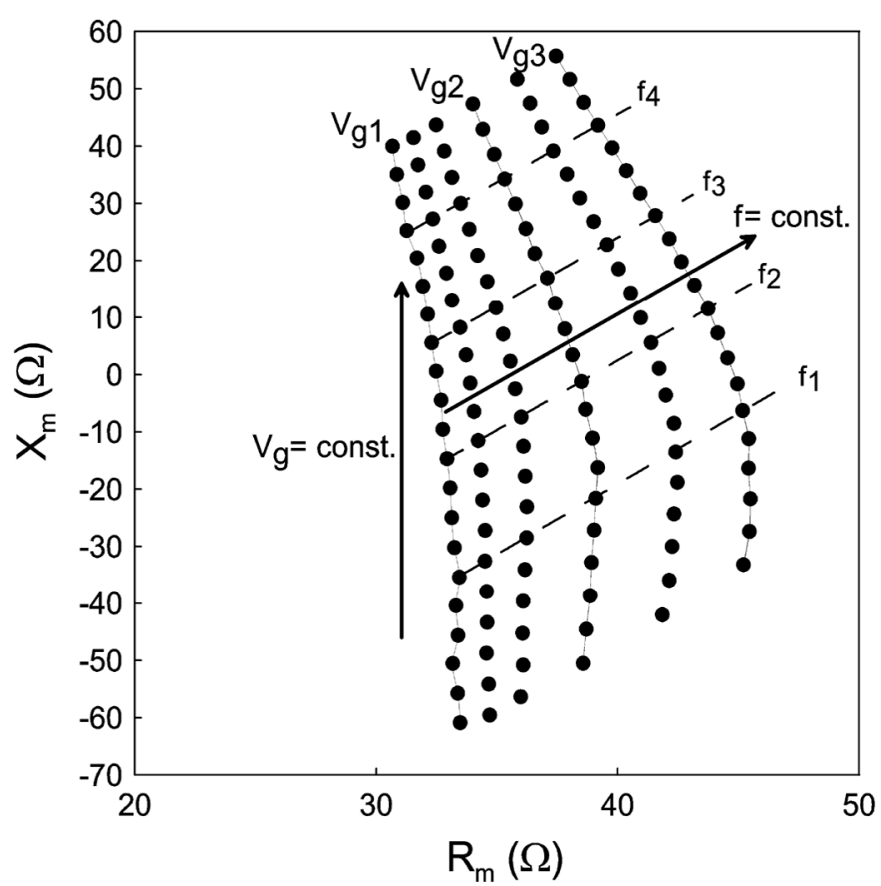

Fig. 4. Resonance measurements at constant voltage and at constant frequency, in the motional impedance plane: straight lines, with slope $m$, for sweeps at constant frequency. PXE5 material.

mean stress $\langle T\rangle$ are more related to the material nonlinearity and allow a characterization nearer to the ceramic properties.

Assuming that the independent magnitude responsible for the nonlinear behavior is the mean stress $\langle T\rangle$ instead of the mean strain $\langle S\rangle$, it will be necessary to use the magnitude $I^{\prime}$, proportional to the mean stress $\langle T\rangle$ (see (A10)), which for a radial resonator corresponds to the use of a dielectric capacitance $C_{0}=C_{0}^{T}[31]$ :

$$
I^{\prime}=\frac{V}{Z^{\prime}}=V\left(\frac{1}{Z}-Y_{0}\right)
$$

where

$$
Y_{0}=j \omega C_{0}^{T}
$$

A very interesting analysis, of great help in understanding the nonlinear behavior near the resonance, can be carried out through the representation of the motional complex impedance $\boldsymbol{Z}_{\boldsymbol{m}}$ in the impedance plane (Fig. 4). It is observed that when the amplitude level at constant frequency increases, the motional resistance $R_{m}$ increases when the losses increase, and the motional reactance $X_{m}$ also increases. In the impedance plane, the points corresponding to an amplitude sweep at constant frequency $f_{1}$ are aligned on a straight line with a slope $m$. For other close frequencies this representation is similar on another straight line nearly parallel to the above line. When the frequency of this second sweep is higher than that of the first sweep, $f_{2}>f_{1}$, it can be observed that the second straight line corresponds to higher reactances $X_{m}$. This 

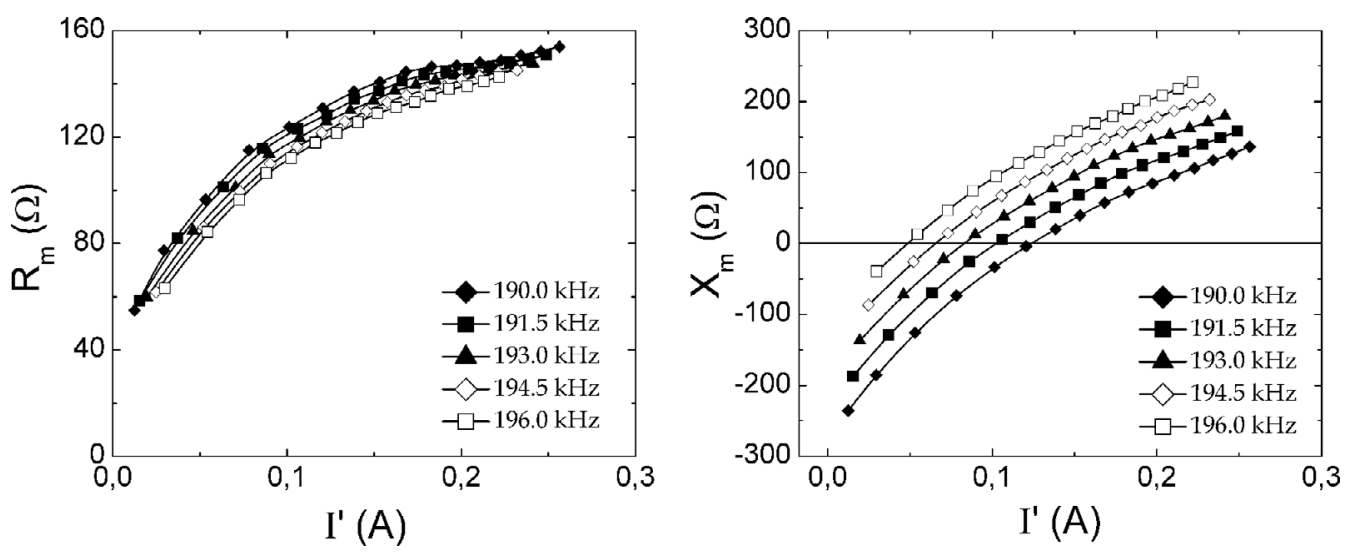

Fig. 5. Motional resistance $R_{m}$ and reactance $X_{m}$ versus the current $I^{\prime}$, for different frequency sweeps. Soft Pz 27 material, from Ferroperm Piezoceramics A/S.

representation shows that the nonlinearity due to the amplitude increase brings about a reactance increase. Thus, in order to reach the resonance, that is to say, $X_{m}=0$, it is necessary to use a lower frequency with a high-level signal: point A at low signal, point B at high signal. One may therefore conclude that by increasing the excitation amplitude, there is equivalence between the decreases or shift frequency of resonance $f_{s}$ and the motional reactance increase $\Delta X_{m}$ [11], a relation previously pointed out in (2).

Fig. 5 shows the motional resistance $R_{m}$ and reactance $X_{m}$ dependences versus the current $I^{\prime}$. It can be observed that, with a good approximation, these curves are parallel for different frequencies. So, the variations $\Delta R_{m}$ and $\Delta X_{m}$ are approximately frequency independent. This result allows us to state that it suffices to perform only one simple amplitude sweep, at a frequency near the resonance, to obtain the laws $R_{m}\left(I^{\prime}\right)$ or $X_{m}\left(I^{\prime}\right)$ in the resonator nonlinear characterization. Moreover, it is always prudent to repeat this amplitude sweep at other close frequencies in order to verify that this dependence is approximately the same and independent of the frequency.

This is a very significant result: The nonlinear behavior depends only on the current $I^{\prime}$, which is proportional to the main stress $\langle T\rangle$, and is frequency independent. In view of this result, it is easy to understand that the nonlinear characterization by means of frequency sweeps at constant voltage or constant current would imply a slower data acquisition, since it would be necessary to make more individual measurements (corresponding to one frequency and one amplitude level) than those carried out in the case of one amplitude sweep at constant frequency. In the latter case, it suffices to perform one sweep at constant frequency and increasing amplitudes.

This result also enables us to draw an appropriate comparison between the three characterization systems (Fig. 6) in the motional impedance plane $X_{m}\left(R_{m}\right)$. In the case of constant voltage, the impedance curve is bent with a great resistance near the resonance, because at these frequencies the total resonator impedance is minimum and the current $I^{\prime}$ is maximum; so the resistance is also maximum. This representation is unable to show that the inde-

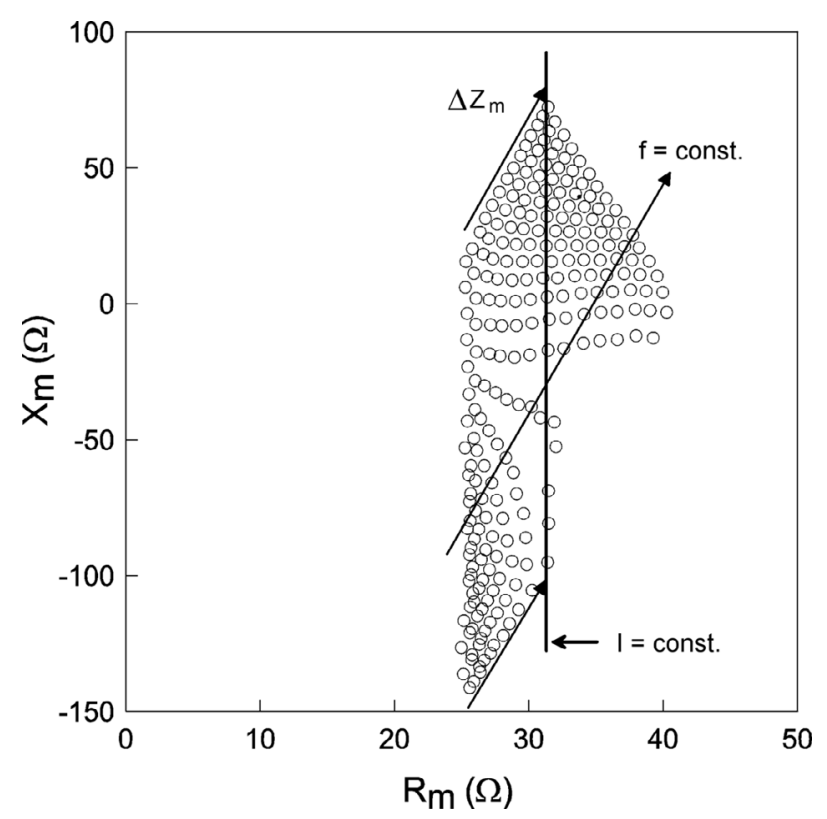

Fig. 6. Measurements in the motional impedance plane: straight lines at constant frequency; vertical straight line for sweep frequencies at constant current. At high level, the impedance increase $\Delta Z_{m}$ is frequency independent.

pendent magnitude is the current $I^{\prime}$. As previously stated, it is necessary to repeat as many sweeps at constant amplitude as points of the nonlinear characterization.

Second, for a characterization at constant current [6], [25], the nonlinearity produces $\Delta R_{m}$ and $\Delta X_{m}$ that are constant throughout the entire sweep, so the curve in this $Z_{m}$ plane is approximately a straight line, parallel to the corresponding one at low signal. The straight line at low signal is displaced $\Delta R_{m}$ horizontally and $\Delta X_{m}$ vertically, quantities that are constant across all of the sweep, since the current $I$ is constant (also $I^{\prime}$ ). The fact that in this case there is no bending of the curve in this impedance plane ensures the disappearance of the hysteresis phenomenon [11], [12].

Finally, when the amplitude sweep is carried out at constant frequency, as proposed in this paper, the measured 


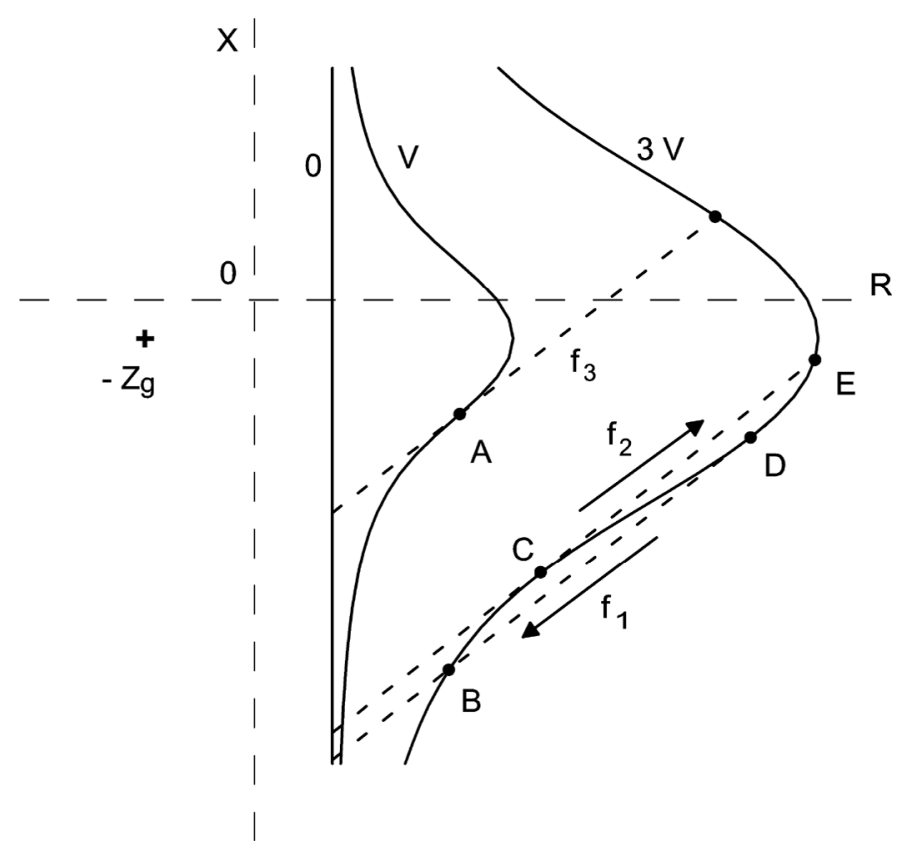

Fig. 7. Curves at constant voltage and straight lines at constant frequency: hysteresis phenomenon in the interval $f_{1}<f<f_{2}$ only for high amplitude level.

points are aligned on a straight line with a slope $m$, as mentioned above. The straight lines are parallel for different frequencies and are longer the closer they are to the resonance $X=0$ at high signal.

\section{B. Hysteresis Phenomenon}

The use of the motional impedance plane $\boldsymbol{Z}_{\boldsymbol{m}}$ also allows us to characterize the hysteresis phenomenon. When the resonator is excited at high signal level, a frequency sweep performed at constant voltage may pass through a different path or points if this sweep is made with increasing or decreasing frequencies (see Fig. 1). Fig. 7 shows [11] that when the slope $m$ of the straight lines at constant frequency is higher than the slope of the curves at constant voltage, $d X_{m} / d R_{m}$, then the intersection between the straight lines and the curves at constant voltage is produced at three points for one frequency (in the interval $f_{1}<f<f_{2}$, in Fig. 7). A point is reached when the sweep is made at increasing frequencies (zone $\mathrm{B}-\mathrm{C}$ ); another when the sweep is made at decreasing frequencies (zone D-E), and the third is unattainable (zone CD) because it is unstable. Thus the slope $m$ characterizes this hysteresis phenomenon completely. At low signal, the hysteresis never appears, because the curve at constant voltage is approximately a straight line. Only when the nonlinearity bends the curve enough, at constant voltage, does the hysteresis appear. The value of this slope $m$ is near $m=2$ for soft PZT ceramics, and very much higher, $m=6$ or 12 , for hard piezoceramics. This result implies that it is in hard materials used in power devices where the hysteresis phenomenon is more likely to be reached.

If the Hirose characterization [6], [25] is used at constant current, this phenomenon does not occur, since the sweep curves remain vertical straight lines and the intersection with the straight lines at constant frequency always gives only one point.

It has been observed that the hysteresis zone always occurs in the lower zone $\left(X_{m}<0\right)$ of the bent curve at constant voltage; that is to say, at frequencies lower than the resonance. The resonance is always reached with decreasing frequencies. When amplitude sweeps are used at constant frequency, a zone of the impedance plane $\boldsymbol{Z}_{\boldsymbol{m}}$ without measured points (see Fig. 6) has also been observed [11], since these points correspond to the abovementioned unattainable points.

It is important to point out that the extreme part of this bent curve, with the maximum resistance $R_{m}$, is found where the current $I^{\prime}$ is also maximum. Generally, it occurs just at resonance, $X=0$. However, this place depends on the amplifier employed. Indeed, the current is maximum when the total reactance, the resonator reactance $X$ plus the amplifier reactance $X_{g}$, is zero, $X+X_{g}=0$. So, this curve maximum at constant voltage is moved vertically a quantity $X=-X_{g}$ due to the amplifier reactance [11]. It is equivalent to moving the origin, and is the cause of the bending on the constant voltage sweep representation, vertically $-X_{g}$ and horizontally $-R_{g}$. This analysis of the experimental system, which includes the generator-amplifier impedance, allows us to avoid the hysteresis phenomenon when it appears. If a series resistance $R_{s}$ is added between the amplifier and the resonator, the bent curve at constant voltage will be less bent and the hysteresis disappears. The curve looks more like a vertical straight line, because the horizontal origin is at $-R_{g}-R_{s}$. This new set-up makes the configuration somewhat similar to that proposed by Hirose with a constant current generator. This system has been verified and used every time that the hysteresis appears. The only problem with this system is that the maximum current reached is lower at resonance.

\section{Elastic Nonlinear Characterization}

The high performance of the above results is clearly shown in Fig. 8 [31]. Fig. 8(a) and (b) shows the resonator total impedance near the resonance [Fig. 8(a)] and near the antiresonance [Fig. 8(b)]. It is observed that when the excitation level increases, the antiresonance is reached from the low level resonance, due to the reactance nonlinear increase. However, all of these curves become parallel straight lines when they are represented in the motional impedance plane $\boldsymbol{Z}_{\boldsymbol{m}}$ [Fig. 8(c)], or when they are analyzed in terms of the current $I^{\prime}$, proportional to the mean stress $\langle T\rangle$ [Fig. 8(d)].

A laser vibrometer enables us to obtain the velocity of a resonator point, so it allows the calculation of the main strain $\langle S\rangle$ in the vibration direction. Thus the dependence of the quotient $D^{\prime} /\langle S\rangle=f(v)$, and the study of the nonlinear electromechanical quotient $D^{\prime} /\langle S\rangle \propto I^{\prime} / v$, between the motional current and the velocity can be obtained when the resonator vibration velocity increases. Generally, 

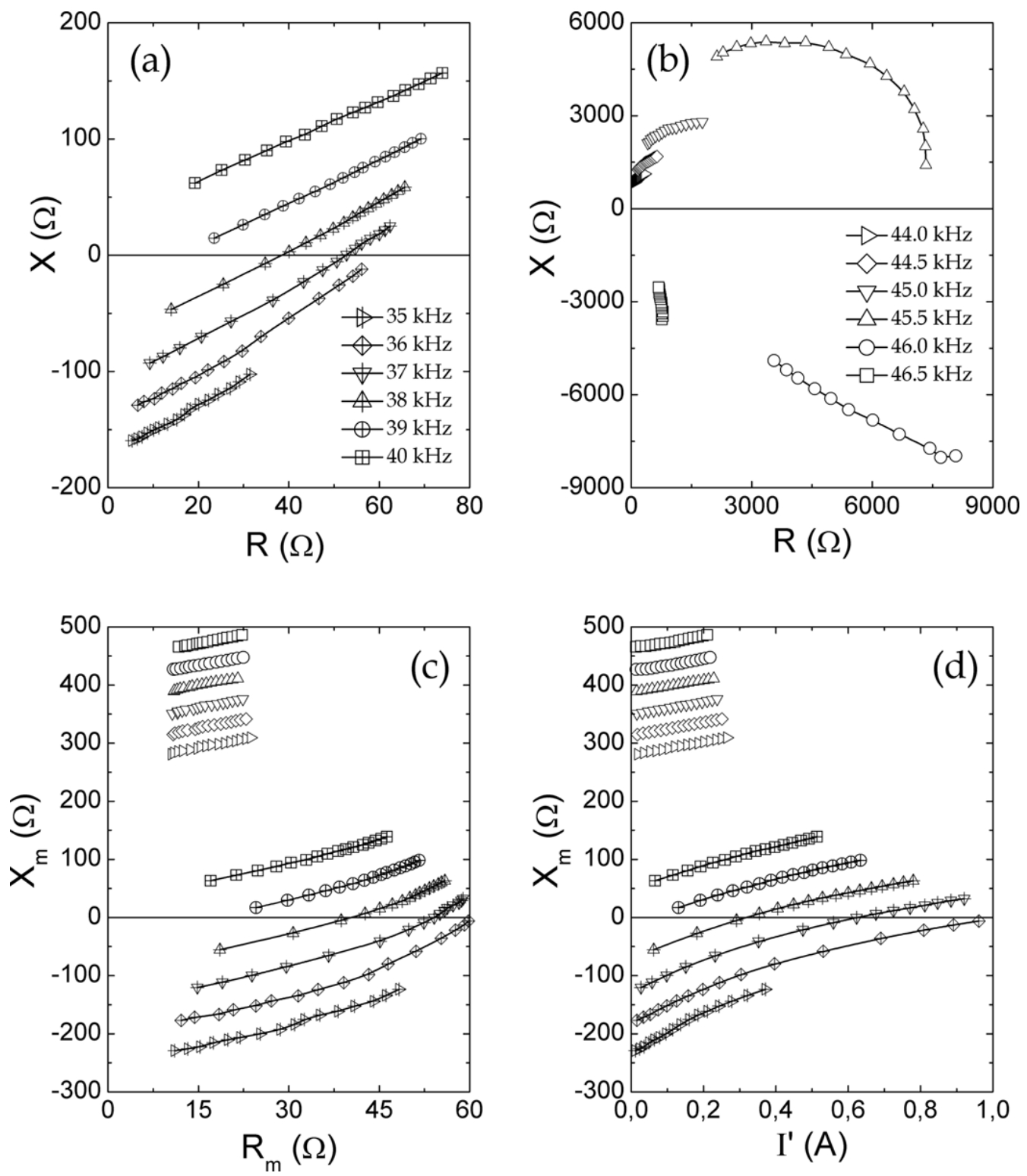

Fig. 8. Resonance (a) and antiresonance (b) measurements at constant frequencies, in the impedance plane; (c) the same measurements in the motional impedance plane; (d) motional reactance versus current $I^{\prime}$. Soft PZT-5A material, from Morgan Matroc Ltd., Southampton, UK.

it has been observed that these quotients are weakly nonlinear in ceramics (Fig. 9).

The representations of the nonlinear motional resistance $R_{m}\left(I^{\prime}\right)$ and motional reactance $X_{m}\left(I^{\prime}\right)$, for different materials show very different dependences. For soft materials, this dependence is shown in Fig. 5 (soft ceramic Pz27, Ferroperm Piezoceramics A/S, Kvistgård, Denmark), and a proportional dependence is verified:

$$
\begin{aligned}
& \Delta R_{m}=r_{1} \cdot I^{\prime}, \\
& \Delta X_{m}=x_{1} \cdot I^{\prime} .
\end{aligned}
$$

For hard ceramic materials, Fig. 10, the dependence versus the current $I^{\prime}$ ceases to be linear and becomes approximately quadratic:

$$
\begin{aligned}
& \Delta R_{m}=r_{2} \cdot I^{\prime 2}, \\
& \Delta X_{m}=x_{2} \cdot I^{\prime 2} .
\end{aligned}
$$

These dependences, either linear or quadratic, have been also observed in the same ceramic materials when a nonlinear dielectric characterization is performed at low frequency (typically at $1 \mathrm{kHz}$ ). In this case, the dependence is between the increase of dielectric permittivity (and the dielectric losses) versus the applied electric field. For soft materials, the nonlinear dielectric behavior shows a dependence on the electric field amplitude $\Delta \varepsilon=\alpha \cdot E_{0}$, in agreement with the Rayleigh model [18], [21], [32].

Finally, the magnitudes $R_{m}, X_{m}, I^{\prime}$, and $v$ must subsequently be modified in order to obtain other magnitudes closer to the constitutive equations and to the ceramic elastic coefficients. To this end, the mechanical losses tangent $\tan \left(\delta_{m}\right)$ is used instead of $R_{m}$, and the shift resonance frequency instead of the motional reactance, by the expressions: 


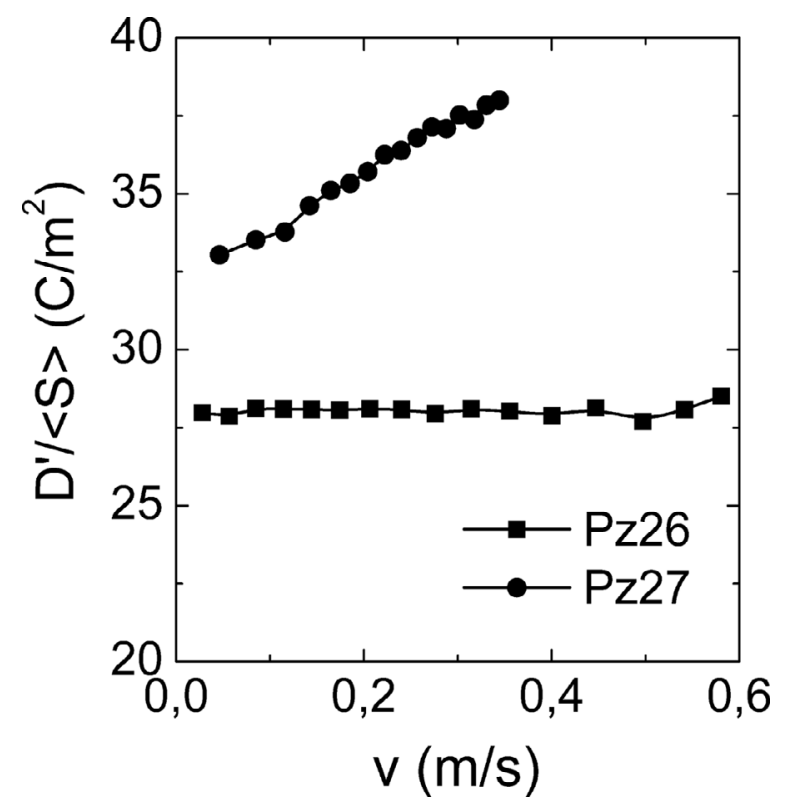

Fig. 9. Dependence of $D^{\prime} /\langle S\rangle$ versus the velocity $v$ of a resonator edge point. Soft $\mathrm{Pz} 27$ and hard $\mathrm{Pz} 26$ materials, from Ferroperm Piezoceramics A/S.

$$
\begin{aligned}
& \frac{\Delta R_{m}}{\bar{Z}}=\Delta \tan \delta_{m}=\Delta\left(\frac{1}{Q_{m}}\right)=F\left(I^{\prime}\right), \\
& \frac{\Delta X_{m}}{\bar{Z}}=-2 \frac{\Delta f}{f_{0}}=F^{\prime}\left(I^{\prime}\right) .
\end{aligned}
$$

The main strain $\langle S\rangle$ can be obtained through the current $I_{m}$ or by laser measurements of $v$. The main stress $\langle T\rangle$ is obtained from $I^{\prime}$. In the case of a disc resonator in the radial mode, the expressions developed in the Appendix are fulfilled.

After obtaining the direct measurements $R, X, I$, and $v$, as well as the modified magnitudes $R_{m}, X_{m}, I^{\prime}, D^{\prime}$, $D_{m}, \Delta f / f$, and $\langle S\rangle$, it is necessary to make some transformations to analyze the elastic and piezoelectric coefficient variations. From the linear elastic coefficient $c_{110}^{P}$ and the decrease of resonant frequency (increase of motional reactance), it is possible to obtain the high signal stiffness $c_{11}^{P}$, assuming that the Poisson ratio $\sigma$ remains constant:

$$
\sqrt{\frac{c_{110}^{P}}{c_{11}^{P}}}=1+\frac{\left(X_{m}-X_{m 0}\right) / \omega}{\frac{d X_{m 0}}{d \omega}}
$$

where $X_{m 0}$ is the low signal motional reactance, $\omega$ is the angular frequency of the measurement amplitude sweep, and $d X_{m 0} / d \omega$ is the linear variation of the motional reactance with the angular frequency.

From the vibration velocity in edge disc point measurements, the mean strain $\langle S\rangle$ (A7), the electric displacement $D_{m}$ (A15), and the piezoelectric coefficients $e_{31}$ and $d_{31}$ can be obtained:

$$
\begin{aligned}
e_{31} & =\frac{D_{m}}{\langle S\rangle}, \\
d_{31} & =\frac{e_{31}}{c_{11}^{P}(1+\sigma)},
\end{aligned}
$$

as well as the mean stress $\langle T\rangle(12)$.

With all of these magnitudes it is possible to analyze these coefficient nonlinear variations with the mean stress, assuming that the Poisson coefficient $\sigma$ remains constant. Fig. 11 shows the dependence between the elastic $c_{11}$ coefficient and the mean stress for two different materials. The relation between the piezoelectric coefficient $d_{31}$ and the elastic $s_{11}$ allows an interpretation of these results, obtaining that the extrinsic coefficient $\Delta d_{31} / \Delta s_{11}$ is higher than the low signal linear $d_{31} / s_{11}$ coefficient [31].

The proposed independent variable is the mean stress $\langle T\rangle$ or the mean strain $\langle S\rangle$. The advantage of the use of the mean stress is that the nonlinear relations are verified both in the resonance and in the antiresonance, but the drawback is the necessary hypothesis to obtain this mean stress: When the stress is obtained from (A12), it is necessary to suppose that the Poisson coefficient $\sigma$ is constant. Otherwise, the use of the mean strain $\langle S\rangle$ as the independent variable is easier, because (A7) allows its direct measurement (it is also possible to obtain the mean strain from (A15) with the hypothesis that $e_{31}^{P}$ is constant, if the velocity vibration has not been measured). In this case, the nonlinear behavior depends weakly on the frequency between resonance and antiresonance.

\section{ViI. Amplitude Sweep at Constant Motional Reactance: Strict Resonance}

A final improvement in the measurement system is proposed. It consists of making the amplitude sweep at constant motional reactance, in the resonance $X_{m}=0$, instead of at constant frequency. To do this, it is necessary to change the frequency and the amplitude in order to ensure the $X_{m}=$ constant condition. As previously described, a similar burst signal is used.

The main advantage of this system is that no hypothesis about the nonlinear behavior being independent from frequency is necessary. It also improves the characterization possibilities and provides a better comparison with other classical nonlinear measurement systems.

In this case, it is necessary for the control computer to know the electric capacitance $C_{0}^{P}$ in order to calculate the electric branch admittance $Y_{0}$. For each measurement, the impedance $\boldsymbol{Z}$ is calculated as well as the motional impedance $\boldsymbol{Z}_{\boldsymbol{m}}$ (8). At the beginning, the low signal measurement must correspond to values of $R_{m}$ and $X_{m}$ near the resonance (Fig. 12, point a). The amplitude increases until $X_{m}>0$ (point b). At this moment the frequency decreases, at constant amplitude (point c), reaching $X_{m}<0$. After the impedance measurement is obtained, the amplitude increases until $X_{m}>0$ again (point d), the process is repeated, decreasing the frequency, and so on. After taking all of these measurements, it is necessary to make the 

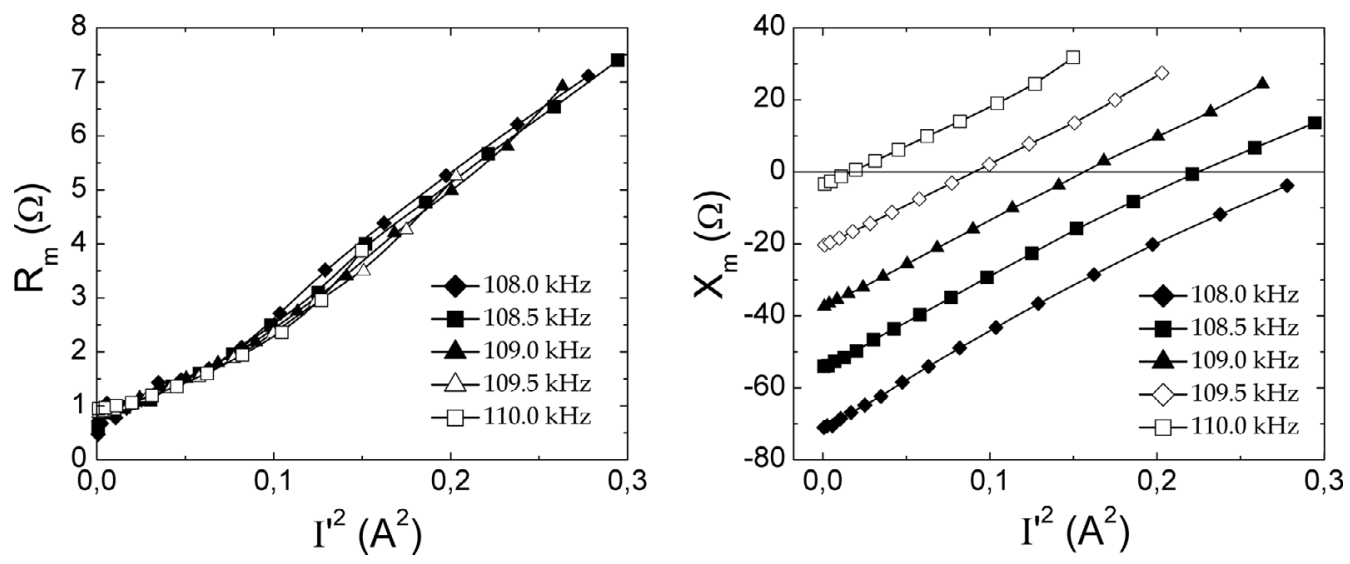

Fig. 10. Motional resistance $R_{m}$ and reactance $X_{m}$ versus the square of current $I^{\prime}$. Hard $\mathrm{Pz} 26$.

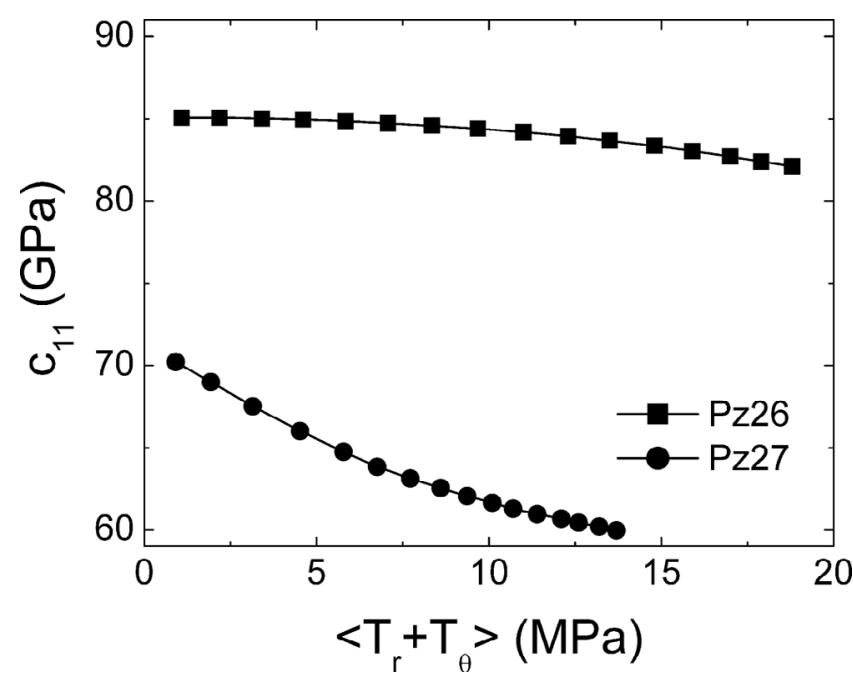

Fig. 11. Stiffness coefficient $c_{11}$ versus the mean stress $\langle T\rangle$. Soft $\mathrm{Pz} 27$ and hard $\mathrm{Pz} 26$ materials.

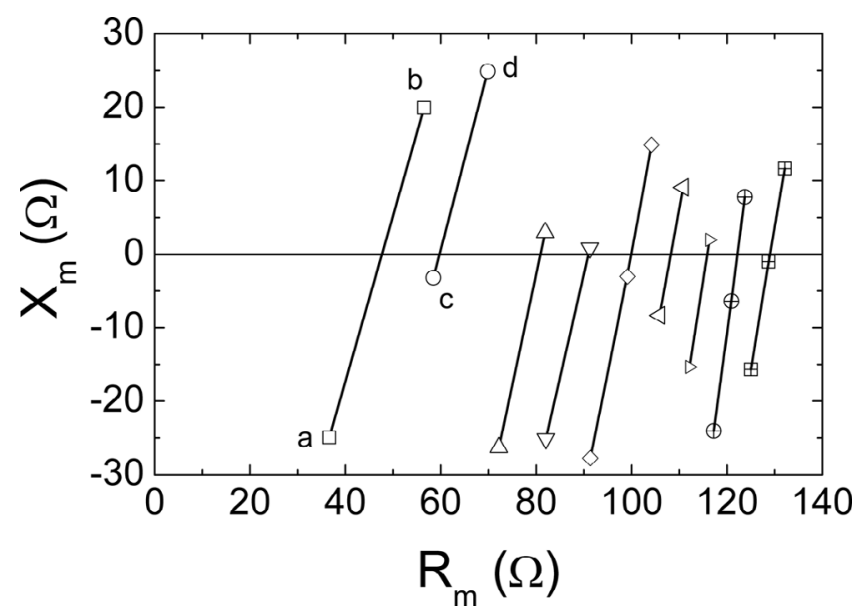

Fig. 12. Measurement points, in the motional impedance plane, to obtain the strict resonance values at $X_{m}=0$ by interpolation. interpolations to obtain for each frequency the amplitude level corresponding to the resonance, $X_{m}=0$. The necessary data to analyze the nonlinear behavior will be the current $I^{\prime}$, the frequency $\omega_{s}$, the resistance $R_{m}$, and the vibration velocity $v$.

This process is necessary to prevent the hysteresis phenomenon, because the interpolation would otherwise be incorrect.

\section{CONCLUSIONS}

An exhaustive study to optimize the high signal resonance measurements in order to allow the nonlinear characterization has been carried out. Burst signal excitations with increasing amplitudes at constant frequency near the resonance have been used. The burst signals are the only ones that prevent the temperature increase typically associated with high signal measurements, which would otherwise distort the results. The proposed systems do not use impedance analyzers, but rather burst excitation signals in short time intervals when the measurement is taken.

The use of the mean stress $\langle T\rangle$ as independent magnitude is proposed for the nonlinear characterization, because it has been verified that it provides a better nonlinear characterization throughout all of the resonanceantiresonance interval. This magnitude could be easily obtained from the current $I^{\prime}$, close to the motional current. The nonlinear characterization shows a dependence on all coefficients - elastic, piezoelectric, and dielectric - with the mean stress amplitude $\langle T\rangle$ in the resonator.

The study through the motional impedance plane is a decisive factor for understanding the nonlinear behavior and for determining the different possible characterization methods. When the frequency-independent condition is verified, the nonlinear characterization is carried out through a single amplitude sweep at constant frequency. Otherwise, a measurement system which always ensures the resonance is also performed.

These measurement types, at constant frequency or at strict resonance, have advantages over the classical systems, which make frequency sweeps at constant voltage or 
constant current by reducing the number of measurement points, since a single amplitude sweep is sufficient.

Together with a numerical simulation that provides the relation between the nonlinear measured laws with the behavior at each resonator point and instant, this system can be of great help in determining high signal phenomena in resonators more precisely.

\section{Appendix A}

\section{Radial Resonance in a Disc}

For a disc of radius $R$ and thickness $t$, verifying the standard conditions [24], [33] $R \gg t$ in radial resonance, the strains $S_{r}$ and $S_{\theta}$ depend only on the radial mechanical displacement $u$. It is possible to define a strain $S$, and a stress $T$, by [19], [24], [33]:

$$
\begin{aligned}
& S=S_{r}+S_{\theta}=u^{\prime}+\frac{u}{r}=\frac{1}{r} \frac{d(u r)}{d r}, \\
& T=T_{r}+T_{\theta} .
\end{aligned}
$$

The constitutive equations may be written as

$$
\begin{aligned}
S & =s_{11}^{E}(1-\sigma) T+2 d_{31} E_{3}, \\
D_{3} & =d_{31} T+\varepsilon_{33}^{T} E_{3}, \\
\sigma & =-\frac{s_{12}^{E}}{s_{11}^{E}}
\end{aligned}
$$

where $\sigma$ is the Poisson coefficient.

To obtain the mean value $\langle X\rangle$ for any magnitude $X$ on the entire disc surface, the calculus of the integral is necessary:

$$
\langle X\rangle=\frac{2}{R^{2}} \int_{0}^{R} X r d r
$$

So, the mean strain value can be an accessible magnitude by measuring the radial vibration movement in a disc edge point, for example, through the radial vibration velocity on a border of the disc $v(R)$ :

$$
\langle S\rangle=\frac{2 u(R)}{R}=\frac{2 v(R)}{R \omega} .
$$

In addition, other accessible magnitudes, which can be calculated from the tension $V$ and current $I$, are the electric field $E$, which is uniform, and the mean electric displacement $\left\langle D_{3}\right\rangle$, related by:

$$
\begin{aligned}
\langle S\rangle & =s_{11}^{E}(1-\sigma)\langle T\rangle+2 d_{31} E_{3}, \\
\left\langle D_{3}\right\rangle & =d_{31}\langle T\rangle+\varepsilon_{33}^{T} E_{3}, \\
\left\langle D^{\prime}\right\rangle & =\left\langle D_{3}\right\rangle-\varepsilon_{33}^{T} E_{3}=d_{31}\langle T\rangle=\frac{I^{\prime}}{j \omega A} .
\end{aligned}
$$

These equations show the relation between the mean stress $\langle T\rangle$ and the current $I^{\prime}$, obtained from the total current $I$ after subtracting the current across the electrical branch, with capacitance $C_{0}^{T}$.
From (A8) and (A9), it is possible to obtain

$$
\begin{aligned}
\left\langle D_{3}\right\rangle & =e_{31}^{P}\langle S\rangle+\varepsilon_{33}^{P} E_{3}, \\
\langle T\rangle & =c_{11}^{E}(1+\sigma)\langle S\rangle-2 e_{31}^{p} E_{3}, \\
e_{31}^{p} & =\frac{d_{31}}{s_{11}^{E}(1-\sigma)}, \\
\varepsilon_{33}^{p} & =\varepsilon_{33}^{T}-\frac{2 d_{31}^{2}}{s_{11}^{E}(1-\sigma)} .
\end{aligned}
$$

where $e_{31}^{P}$ is the planar piezoelectric coefficient.

From (A11), the mean $\left\langle D_{m}\right\rangle$ value can be obtained,

$$
\left\langle D_{m}\right\rangle=\left\langle D_{3}\right\rangle-\varepsilon_{33}^{P} E_{3}=e_{31}^{P}\langle S\rangle=\frac{I_{m}}{j \omega a} .
$$

So, the motional current $I_{m}$ can be calculated from the total current $I$ by subtracting the current across the electrical branch, with capacitance $C_{0}^{P}$. This motional current is proportional to the mean strain $\langle S\rangle$. The capacitance $C_{0}^{P}$ corresponds to the dielectric planar constant $\varepsilon_{33}^{P}[24]$, obtained after the radial resonance and before the thickness resonance.

\section{REFERENCES}

[1] H. F. Tiersten, "Analysis of nonlinear resonance in thicknessshear and trapped-energy resonators," J. Acoust. Soc. Amer., vol. 59, no. 4, pp. 866-878, 1976.

[2] J. Nosek, "Neleneárí elektrický náhradní obvod piezoelektrického rezonátoru," Slaboproudý obzor, vol. 45, pp. 133-137, 1984. (in Czech)

[3] M. Planat and D. Hauden, "Nonlinear properties of bulk and surface acoustic waves in piezoelectric crystals," in Piezoelectricity. G. W. Taylor, J. J. Gagnepain, T. R. Meeker, T. Nakamura, and L. A. Shuvalov, Eds. New York: Gordon and Breach, 1985, pp. $277-296$.

[4] H. Beige and G. Shmidt, "Electromechanical resonances for investigating linear and nonlinear properties of dielectrics," in Piezoelectricity. G. W. Taylor, J. J. Gagnepain, T. R. Meeker, T. Nakamura, and L. A. Shuvalov, Eds. New York: Gordon and Breach, 1985, pp. 93-103.

[5] R. Holland and E. P. Eernisse, "Accurate measurement of coefficients in a ferroelectric ceramic," IEEE Trans. Sonics Ultrason., vol. SU-16, no. 4, pp. 173-181, 1969.

[6] K. Uchino, Piezoelectric Actuators and Ultrasonic Motors. H. L. Tuller, Ed. Norwell, MA: Kluwer Acad. Pub., 1997.

[7] S. Priya, D. Viehland, A. Vazquez Carazo, J. Ryu, and K. Uchino, "High-power resonant measurements of piezoelectric materials: Importance of elastic nonlinearities," J. Appl. Phys., vol. 90, no. 3, pp. 1469-1479, 2001.

[8] D. Guyomar, N. Aurelle, C. Richard, P. Gonnard, and L. Eyraud, "Non linearities in Langevin transducers," in Proc. IEEE Ultrason. Symp., 1994, pp. 925-928.

[9] H. F. Tiersten and A. Ballato, "Nonlinear extensional vibrations of quartz rods," J. Acoust. Soc. Amer., vol. 73, no. 6, pp. 2022 2033, 1983.

[10] S. Tashiro, M. Ikehiro, and H. Igarashi, "Influence of temperature rise and vibration level on electromechanical properties of high-power piezoelectric ceramics," Jpn. J. Appl. Phys., vol. 36, pp. 3004-3009, 1997.

[11] R. Pérez and A. Albareda, "Analysis of non-linear effects in a piezoelectric resonator," J. Acoust. Soc. Amer., vol. 100, no. 6, pp. 3561-3570, 1996.

[12] A. Albareda, P. Gonnard, V. Perrin, R. Briot, and D. Guyomar, "Characterization of the mechanical nonlinear behavior of piezoelectric ceramics," IEEE Trans. Ultrason., Ferroelect., Freq. Contr., vol. 47, no. 4, pp. 844-853, 2000. 
[13] S. Li, W. Cao, and L. E. Cross, "The extrinsic nature of nonlinear behavior observed in lead zirconate titanate ferroelectric ceramic," J. Appl. Phys., vol. 69, pp. 7219-7224, 1991.

[14] Q. M. Zhang, H. Wang, N. Kim, and L. E. Cross, "Direct evaluation of domain wall and intrinsic contributions to the dielectric and piezoelectric response and their temperature dependence on lead zirconate titanate ceramics," J. Appl. Phys., vol. 75, pp. 454-459, 1994

[15] R. Herbiet, U. Robels, H. Dederichs, and G. Arlt, "Domain wall and volume contributions to material properties of PZT ceramics," Ferroelectrics, vol. 98, pp. 107-121, 1989.

[16] D. Hall and P. Stevenson, "High field dielectric behavior of ferroelectric ceramics," Ferroelectrics, vol. 228, pp. 139-158, 1999.

17] G. Robert, D. Damjanovic, N. Setter, and A. V. Turik, "Preisach modeling of piezoelectric nonlinearity in ferroelectric ceramics," J. Appl. Phys., vol. 89, pp. 5067-5074, 2001.

[18] D. Damjanovic and M. Demartin, "Contribution of the irreversible displacement of domain walls to the piezoelectric effect in barium titanate and lead zirconate titanate ceramics," $J$. Phys.: Condens. Matter, vol. 9, pp. 4943-4953, 1997.

[19] R. Pérez, A. Albareda, J. E. García, J. Tiana, E. Ringgaard, and W. W. Wolny, "Extrinsic contribution to the non-linearity in a PZT disc," J. Phys. D: Appl. Phys., vol. 37, pp. 2648-2654, 2004.

[20] J. E. García, R. Pérez, and A. Albareda, "High electric field measurement of dielectric constant and losses of ferroelectric ceramics," J. Phys. D: Appl. Phys., vol. 34, pp. 3279-3284, 2001.

[21] D. Hall, "Rayleigh behaviour and the threshold field in ferroelectric ceramics," Ferroelectrics, vol. 223, pp. 319-328, 1999.

[22] A. Albareda, R. Pérez, J. L. Villar, E. Minguella, and J. A. Gorri, "Intermodulation measurement of nonlinearities in piezoceramic resonators," Rev. Sci. Instrum., vol. 68, no. 8, pp. 3143-3149, 1997.

[23] A. Albareda, J. H. Kayombo, and J. A. Gorri, "Nonlinear direct and indirect third harmonic generation in piezoelectric resonators by the intermodulation method," Rev. Sci. Instrum., vol. 72 , no. 6 , pp. $2742-2749,2001$.

[24] European Standard, "Piezoelectric properties of ceramic materials and components-Part 3: Methods of measurement-High power," CENELEC, 50324-3, 2002

25] S. Hirose, S. Takahashi, K. Uchino, M. Aoyagi, and Y. Tomikawa, "Measuring methods for high-power characteristics of piezoelectric materials," in Proc. Mater. Res. Soc. Symp. vol. 360, 1995, pp. 15-20.

[26] A. Albareda, J. A. Casals, R. Pérez, and F. Montero de Espinosa, "Nonlinear measurements of piezocomposite transducers with burst excitation," in Proc. IEEE Int. Symp. Appl. Ferroelectr., 2001, pp. 979-982.

[27] J. A. Casals, A. Albareda, R. Pérez, J. E. García, E. Minguella, and F. Montero de Espinosa, "Non-linear characterization with burst excitation of 1-3 piezocomposite transducers," Ultrasonics, vol. 41 , no. 4 , pp. $307-311,2003$.

[28] A. Albareda, J. A. Casals, R. Pérez, and F. Montero de Espinosa, "Nonlinear measurements of high-power 1-3 piezo-airtransducers with burst excitation," Ferroelectrics, vol. 273, pp. 47-52, 2002.

[29] S. Takahashi, Y. Sasaki, M. Umeda, K. Nakamura, and S. Ueha, "Nonlinear behavior in piezoelectric ceramic transducers," in Proc. IEEE Int. Symp. Appl. Ferroelectr., 2001, pp. 11-16.

[30] M. Umeda, K. Nakamura, and S. Ueha, "The measurement of high power characteristics for a piezoelectric transducer based on the electrical transient response," Jpn. J. Appl. Phys., vol. 37, pp. 5322-5325, 1998.

[31] A. Albareda, R. Pérez, J. E. García, and D. A. Ochoa, "Nonlinear elastic phenomena near the radial antiresonance frequency in piezoceramic discs," J. Electroceram., 2007.

[32] B. Andersen, E. Ringgaard, T. Bove, A. Albareda, and R. Pérez, "Performance of piezoelectric ceramic multilayer components based on hard and soft PZT," in Proc. Actuators'00, 2000, pp. 419-422.

[33] "IEEE Standard on Piezoelectricity," ANSI/IEEE Std 1761987. IEEE: New York, 1988.

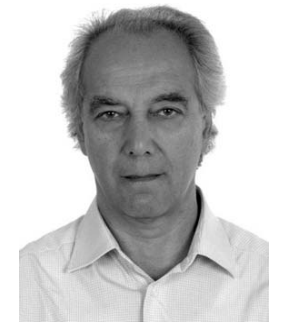

Alfons Albareda was born in Barcelona, Spain, in 1948. He received the Maitrise degree in physics in 1971 and the Ph.D. degree in physics in 1975 from the University Paul Sabatier (Toulouse, France) and the Ph.D. degree in physics from the University of Barcelona in 1982. Since 1977, he has been at the Technical University of Catalonia, Barcelona, Spain, in the Department of Applied Physics, where he has been teaching physics at the School of Telecommunications Engineers.

Since 1989 he has been working on piezoceramic devices and measurement systems, especially in power applications and nonlinear characterization. He is a Spanish member in the European Standardization of high level characterization of piezoceramics. His recent research is in the domain of the piezoceramic materials microstructure, by elastic, dielectric, and piezoelectric nonlinear measurements and by low temperature characterization.

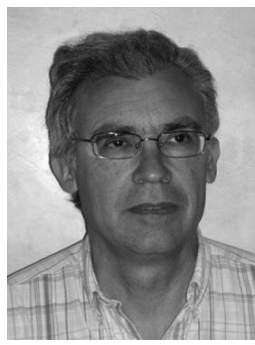

Rafel Pérez was born in Sabadell, Spain, in 1948. He studied physical sciences at Barcelona University from 1965 to 1970 , and received the Ph.D. degree in 1983 at the same university.

He is working at the Technical University of Catalonia, at the Applied Physics Department, and he teaches physics, acoustics, and dielectric and ferroelectric materials at the Telecommunication Engineering School of Barcelona. His research fields include ferroelectric switching, nonlinear dielectric and piezoelectric behavior, and nonlinear piezoelectric resonators.

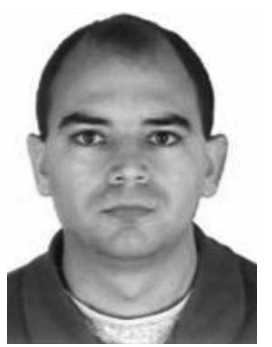

Jorge A. Casals was born in Holguín, Cuba, in 1969. He received the physics degree from the University of Oriente, Cuba, in 1994, and the Ph.D. degree in physics from the Polytechnic University of Catalonia, Barcelona, Spain, in 2005.

Since 1994 to 1997 he worked as a teacher assistant in the Physics Department and during 1998 in the Industrial Engineering Department of the University of Holguin, Cuba. Since 2002 he has been at the Polytechnic University of Catalonia, in the Department of Physics and Nuclear Engineering, where he has been teaching physics at the College of Industrial Engineering of Terrassa, Barcelona. His current research interests include power applications and nonlinear characterization of piezoceramic and piezocomposites devices.

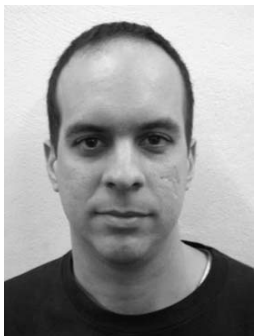

Jose E. García was born in Holguin, Cuba, in 1974 . He received the physics degree from the University of Oriente, Cuba, in 1996 and $\mathrm{Ph} . \mathrm{D}$. degree in applied physics from the Technical University of Catalonia in 2003. He is currently a lecturer at the Department of Applied Physics at the Technical University of Catalonia. His current research interests in ferroelectric materials include dielectric and piezoelectric properties of piezoelectric ceramics. 


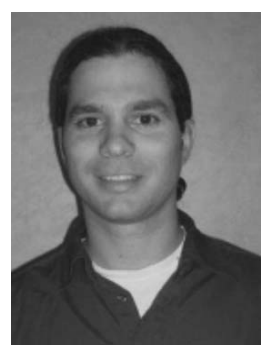

Diego A. Ochoa was born in Havana, Cuba, in 1977. He received the physics degree from the Universidad de La Habana, Cuba, in 2001, and the D.E.A. in applied physics and science simulation from the Universitat Politecnica de Catalunya (UPC), Spain, in 2006. He is currently carrying out research at the UPC to obtain the Ph.D. degree in applied physics. His research area concerns piezoelectric materials, microstructure, and nonlinear properties. 